\title{
Choice of Local Therapy in Children With Ewing Sarcoma
}

\author{
GAURI KAPOOR AND SANDEeP JAIN \\ Department of Pediatric Hematology Oncology, Rajiv Gandhi Cancer Institute and Research Centre, Delhi. \\ kapoor.gauri@rgcirc.org
}

$\mathrm{E}$ wing sarcoma is the second most common bone cancer in children and adolescents and constitutes $40 \%-45 \%$ of malignant bone tumors in this group. Consecutive clinical trials have clearly established the essential role of multidrug chemotherapy and local control measures in curative strategies for this disease. This combined modality approach has led to improvement in long-term disease-free survival to about $70 \%$ in patients with localized or nonmetastatic Ewing sarcoma. Review of published data shows that in Ewing sarcoma, local tumor control can be achieved with either surgery or radiotherapy (RT) or a combination of both. Furthermore, the trials have systematically assessed the importance of sequence and timing of local therapy on outcome. Current data recommends timing for local control to be 10-12 weeks after neo-adjuvant chemotherapy, and delay beyond 16 weeks has been shown to negatively impact survival [1].

Choice of local control modality (surgery, surgery combined with radiotherapy, or radiotherapy alone) and its impact on clinical outcomes such as survival and relapse in patients with non-metastatic Ewing sarcoma is the subject addressed by Zhu, et al. [2] in their article in this issue of the journal. In the absence of randomized trials that directly compare these modalities, the authors have attempted an indirect statistical approach using network meta-analysis to answer this question.

Ewing sarcoma is well known to be a radiosensitive tumor hence both surgery and RT represent effective local treatment modalities. However, recent trends for local management in Ewing sarcoma have favored the surgical approach. This trend may be attributed not only to availability of better surgical techniques, but is also based on reports of increased incidence of local failure rates with RT. These studies report three times higher local relapses in patients treated with radiation alone (failure rate of $30 \%$ ) as compared to patients treated with surgery alone where the rates were less than or equal to $10 \%$ [3-6]. However, we need to interpret these results with caution, as these studies are retrospective and non-randomized and therefore susceptible to inherent selection bias. For example, it has been observed that cases where complete surgical excision was possible had better survival than those treated with RT. On the other hand, tumors that were not amenable to surgical excision and hence treated by radiotherapy alone were often associated with unfavorable features like large volume, central axis location or neurovascular involvement.

Surgery does offer certain advantages; it provides an opportunity to assess response to neo-adjuvant chemotherapy, reassess disease status and reduces the risk of second malignant neoplasms associated with RT. Therefore, the increasing use of surgery as local control modality has led to re-evaluation of the indications for radiotherapy. It is important to remember that surgery and RT are complimentary modalities in the management of Ewing sarcoma, not competitive.

Radiotherapy techniques and indications for Ewing sarcoma have evolved in the last few decades. Definitive radiotherapy is indicated for tumors that are considered unresectable (like sacral tumors crossing midline) or if the morbidity associated with surgery is deemed too high. It is generally agreed that adjuvant radiotherapy (combination of surgery and RT) should be given to patients with positive or very close margins, although various series have used differing cut-offs for amounts of viable tumor in the excised specimen. For others, there are conflicting views on integrating radiotherapy pre-operatively and postoperatively. Euro-Ewing-2012 radiotherapy guidelines recommend postoperative RT in the following clinical situations: if all tissues involved by the pre-chemotherapy tumor volume have not been surgically excised (as often seen in pelvic and sacral sarcomas) or if the histological response to pre-operative chemotherapy is poor $(<90 \%$ necrosis) despite presence of negative surgical margins [7]. Other indications of postoperative RT include a displaced pathological fracture, Askin tumor presenting with pleural effusion and spinal/paraspinal disease where wide surgical excision is unlikely. There are emerging data that show benefit of pre-operative radiotherapy in certain specific clinical situations. These include Ewing sarcoma located at sites like the pelvis or rib, where it allows better delineation of tumor volume and also when the volume of treatment would be smaller than if administered post-operatively. 
Newer techniques like Proton beam radiotherapy have recently been used in patients with Ewing sarcoma. Unlike the standard photon radiotherapy, with the use of proton beam one can minimize radiotherapy to adjacent normal structures while maintaining full dose to the tumor. This technique would therefore be greatly advantageous in patients where the tumor is in close proximity to critical structures like the eye or spinal cord, as well as for young children receiving curative treatment; wherein it would reduce the risk of radiation-induced second malignancy. Hence, we observe that both surgery and radiotherapy have their relative merits, but due to biases in patient selection the conclusions may be obscured.

In an ideal situation, local treatment should be individualized taking into consideration various factors based on patient characteristics, the likely benefit and harm of the treatment modality and preference of the patient. The selection strategy should be planned with the goal of optimizing local tumor control while minimizing therapy related adverse effects.

In conclusion, these decisions about local therapy are usually complex and should preferably be made by a bone sarcoma or musculoskeletal multidisciplinary team that includes surgical, radiotherapy and pediatric oncologists, together with the family. The published study [2] adds to the evidence-base for making informed decisions by such teams.

Competing interests: None; Funding: None.

\section{REFERENCES}

1. Lin TA, Ludmir EB, Liao KP, McAleer MF, Grosshans DR, McGovern SL, et al. Timing of local therapy affects survival in Ewing sarcoma. Int $J$ Radiat Oncol Biol Phys. 2019;104:127-136.

2. Zhu H, Li Y, Xie X, Zhang S, Xue Y, Fan T. Efficacy of local control strategies for ewing sarcoma after neoadjuvant chemotherapy: A network meta-analysis. Indian Pediatr. 2020;57:527-32.

3. Bacci G, Forni C, Longhi A, Ferrari S, Donati D, Paolis M, et al. Long term outcome for patients with non metastatic Ewing's sarcoma treated with adjuvant and neoadjuvant chemotherapies. 402 patients treated at Rizzoli between 1972 and 1992. European J Cancer. 2004;40:73 83.

4. Donaldson SS, Torrey M, Link MP, Glicksman A, Gilula L, Laurie $\mathrm{F}$, et al. A multidisciplinary study investigating radiotherapy in Ewing's sarcoma: End results of POG \#8346. Int J Radiat Oncol Biol Phys. 1998;42:125 35.

5. Krasin MJ, Rodriguez Galindo C, Billups CA, Davidoff AM, Neel MD, Merchant TE, et al. Definitive irradiation in multidisciplinary management of localized Ewing sarcoma family of tumors in pediatric patients: Outcome and prognostic factors. Int J Radiat Oncol Biol Phys. 2004;60: 8308.

6. Schuck A, Ahrens S, Paulussen M, Kuhlen M, Konemann $\mathrm{S}$, Rube C, et al. Local therapy in localized Ewing tumors: Results of 1058 patients treated in the CESS 81, CESS 86, and EICESS 92 trials. Int J Radiat Oncol Biol Phys.2003; 55:168 77 .

7. Gerrand C, Athanasou N, Brennan B, Grimer R, Judson I, Morland $\mathrm{B}$, et al. UK guidelines for the management of bone sarcomas. Clin Sarcoma Res. 2016; 6:7-27. 